\title{
Measuring Group Velocity in Seismic Noise Correlation Studies Based on Phase Coherence and Resampling Strategies
}

\author{
Martin Schimmel, Eleonore Stutzmann, and Sergi Ventosa
}

\begin{abstract}
Seismic noise cross correlation studies are of increasing importance in the seismological research community due to the ubiquity of noise sources and advances on how to use the seismic noise wave field for structural imaging and monitoring purposes. Stacks of noise cross correlations are now routinely used to extract empirical Green's functions between station pairs. In regional and global scale studies, mostly surface waves are extracted due to their dominance in seismic noise wave fields. Group arrival times measured from the timefrequency representation of frequency dispersive surface waves are further used in tomographic inversions to image seismic structure. Often, the group arrivals are not clearly identified or ambiguous depending on the signal and noise characteristics. Here, we present a procedure to robustly measure group velocities using the time-frequency domain phase-weighted stack (PWS) combined with data resampling and decision strategies. The time-frequency PWS improves signal extraction through incoherent signal attenuation during the stack of the noise cross correlations. Resampling strategies help to identify signals robust against data variations and to assess their errors. We have gathered these ingredients in an algorithm where the decision strategies and tuning parameters are reduced for semiautomated processing schemes. Our numerical and field data examples show a robust assignment of surface-wave group arrivals. The method is computational efficient thanks to an implementation based on pseudoanalytic frames of wavelets and enables processing large amounts of data.
\end{abstract}

Index Terms-Group velocities, seismic noise, seismology, surface waves. their efficiency for imaging, many studies have focused on the measurement of surface-wave dispersion (see [3]-[11]; among others). These measurements are usually obtained through a time-frequency representation (TFR) of the data based on the multiple filter technique (MFT) or the moving window analysis [3].

Surface waves have different phase and group velocities [2], [7], [12]. The phase velocity is the speed of each individual wave while the group velocity is the speed of the wave group. The group arrival time is thus related to the propagation of wave energy and, therefore, identified as an energy maximum. Its identification can be difficult due to the presence of other seismic waves through scattering, multipathing, wave type conversions, and other signals and noise.

Group and phase-dispersion studies have been conducted traditionally for earthquake or active source data (human generated sources as explosions, vibrators, weight drop, among others) [12]-[14]. Since the recent last decade [15], [16], the importance of ambient seismic noise imaging studies has been continuously growing in the seismological and geoscientific community (see [17]-[21] among many others), mainly, due to progress on how to use the ubiquitous noise wave fields for imaging purposes. The key difference between noise studies and their corresponding earthquake or active source studies lies in the data acquisition and procedures to extract signal waveforms, often followed by more traditional inversion approaches.

The signal extraction from noise is based on interferometric principles [22], conventionally accomplished through crosscorrelating sequences of simultaneous noise recordings from two stations and subsequent stacking of the resulting cross correlations. If the noise wave field is sufficiently well balanced with respect to the propagation direction of the constituent waves, then empirical interstation Green's functions (EGFs) can be extracted from the noise as theoretically shown using different approaches [23]-[29]. For ambient noise studies, from local-to-global scales, these EGFs contain mainly surface waves due to their dominance over body waves in noise at the frequencies usually considered $(<1 \mathrm{~Hz})$ [30]-[33]. These surface waves can be understood as waves generated at one of the stations (virtual source) and recorded at the other station.

The primary goal of this contribution is to present a new strategy for a robust and semiautomated estimation of seismic group arrival times or group velocities. Our approach differs from other existing techniques, which essentially implement the MFT as described in [3] and [4] (as implemented in the Computer Programs in Seismology package of [11] and 
other packages), by including the stack of cross correlations to estimate group maxima robustly. Furthermore, incoherent noises are attenuated through their low phase coherence using the time-frequency phase-weighted stack (tf-PWS) [34]. In Section II, we present the main ingredients of this method, briefly outlining the underlying theory and how these ingredients are adapted and combined to measure group velocities. Then, the method is tested using theoretical and field data, illustrating the performance, benefits, and limitations.

\section{Material And Methods}

Our main goal is the robust extraction of surface-wave group velocities from EGFs. The input of our approach is the ambient noise cross correlations before stacking and the ingredients of the method are outlined in the following.

\section{A. Green's Function Retrieval}

It has been shown in the different theoretical derivations [23]-[29] that EGFs can be extracted from stacks of seismic noise cross correlations. The cross correlations identify waves recorded by two stations. Ideally, in a system with equipartitioned waves, i.e., where wave energy is balanced as a function of travel direction, and in the presence of a significant number of waves, the noise cross correlations retain signals, which add constructively to the EGF. All other features (including the cross correlation cross terms [35]) are attenuated or canceled out through destructive summation. In practice, the EGF for a pair of stations is computed by cutting the continuous noise recordings into many data sequences, which are then cross-correlated and stacked. Large amplitude signals, as from earthquakes or localized noise sources, usually deteriorate the EGF and may even inhibit an EGF retrieval [17], [36]. Therefore, large data volumes are preprocessed to balance the amplitudes of the noise recordings in the time and frequency domain. Different strategies of amplitude normalization exist [17]. Note that amplitude balancing is not required for the phase cross correlation (PCC) [37], as shown in [36].

\section{B. Analytic Signal and Phase Coherence}

Reference [36] shows that the EGF retrieval can be improved using phase coherence based on analytical signal theory. In essence, the time series $u(t)$ is transformed into the complex domain through computing their analytic signal $s(t)=u(t)+i H[u(t)]$, where $H[u(t)]$ is the Hilbert transform of $u(t)$. The exponential form $s(t)=a(t) \exp (i \Phi(t))$ provides the envelope $a(t)$ and the instantaneous phase $\Phi(t)$. The usual implementation involves two Fourier Transforms: $s(t)=\operatorname{IFT}\left[u^{a}(\omega)\right]$ and $u(\omega)=\mathrm{FT}[u(t)]$, where FT and IFT stand for the forward and inverse Fourier transforms with $u^{a}(\omega)=2 u(\omega)$ for $\omega>0, u^{a}(\omega)=u(\omega)$ for $\omega=0$, and $u^{a}(\omega)=0$ for $\omega<0$.

Phase coherence refers to signals with the same waveforms and, consequently, the same instantaneous phase $\Phi(t)$. The phase coherence [38] is quantified through the summation of the envelope-normalized analytic signals

$$
c(t)=\left|\frac{1}{J} \sum_{j=1}^{J} e^{i \Phi_{j}(t)}\right|^{v}
$$

where the index $j$ labels the $J$ traces (here noise cross correlograms) used in the analysis. $c(t)$ is a time-dependent coherence measure of the degree of constructive summation, which consists of real numbers that range from 0 to 1 , where 1 means that all $N$ signals are completely coherent at time $t$. The exponent $v$ tunes the sensitivity of the measure being $v=2$ an excellent default value. The analytic coherence measure $c(t)$ is the weight of the time domain PWS strategy presented in [38]. It basically down weights signals that are less coherent, independently of their amplitudes. That is, the phase coherence weight $c(t)$ is amplitude unbiased, which permits the detection of coherent weak-amplitude signals masked by other larger amplitude noise.

\section{Time-Frequency Phase-Weighted Stacking}

The phase coherence concept has been extended to the time-frequency domain to improve data adaptation [34]. For this purpose, the PWS method is working with the TFR of the data. The corresponding coherence weights $c(t, f)$ are, therefore, determined as a function of time and frequency to account for nonstationarities in time and frequency. In principle, any TFR, which provides analytic signals, can be used for the tf-PWS. The best results are expected using a multiresolution approach where the window length for timefrequency localization depends on frequency, as it is the case for the wavelet transform and S-transform (ST) [39]. The ST is a continuous wavelet transform written using the notation of a windowed Fourier transform [40] to employ the more physically intuitive concept of frequency in place of scale. If $S_{j}(\tau, f)$ is the TFR of the $j$ th trace obtained using the $\mathrm{ST}$, then the time-frequency phase coherence $c(\tau, f)$ can be written as

$$
c(\tau, f)=\left|\sum_{j=1}^{J} \frac{S_{j}(\tau, f) e^{i 2 \pi f \tau}}{\left|S_{j}(\tau, f)\right|}\right|^{\nu} .
$$

The tf-PWS is then obtained through a matrix multiplication

$$
S_{\mathrm{pws}}(\tau, f)=c(\tau, f) S_{l s}(\tau, f)
$$

where $S_{l s}(\tau, f)$ is the ST of the linear stack (LS) of all traces.

Here, we use the wavelet transform to implement the timefrequency expansion [40] due to its much lower computational costs and redundancy, key elements also to improve the computational efficiency of the subsequent resampling strategy to find robust group arrivals. We perform a time-scale decomposition using discretized frames of wavelet to approximate the continuous wavelet transform (see [41] for a comparison between discrete and continuous wavelet transforms). For this task, we opt for the complex Morlet as mother wavelet, since it approximates an analytic wavelet with an optimal timefrequency resolution. This wavelet writes as a modulated Gaussian

$$
\psi(t)=\pi^{-1 / 4} e^{i \omega_{0} t} e^{-t^{2} / 2}
$$

centered at the frequency $\omega_{0}$. A standard choice of $\omega_{0}=$ $\pi(2 / \ln 2)^{1 / 2}$ makes the amplitude of the side lobes equal to half of the main lobe. 
The continuous wavelet transform [42] of a signal $x(t)$ is given by the inner products with a collection of wavelets

$$
x(\tau, \lambda)=\left\langle x, \psi_{\tau, \lambda}\right\rangle=\int_{-\infty}^{\infty} x(t) \psi_{\tau, \lambda}^{*}(t) d t
$$

where $\tau$ is delay or lag-time and $\lambda$ is scale. The collection of wavelets $\psi_{\tau, \lambda}$ is a set of zero-mean energy-normalized functions generated through scaling and translation operations, $\psi_{\tau, \lambda}(t)=\lambda^{-1 / 2} \psi\left(\lambda^{-1}(t-\tau)\right)$. Therefore, the frequency resolution is proportional to $1 / \lambda$ and the time resolution proportional to $\lambda$.

Frames of wavelets allow us to sample the time-scale domain according to the actual time-frequency resolution, and thus implement the continuous wavelet transform efficiently and accurately, without losing freedom in the choice of the mother wavelet. We specifically discretize scale as $\lambda=2^{j+v / V}$ and delay as $\tau=2^{j} b_{0}$. Index $j \in Z$ is the octave, $v \in$ $[0, \ldots, V-1]$ is the voice, and $b_{0}$ is the sampling period at scale zero, $j=v=0$. The number of samples used in the time-scale domain with respect to the time domain increases by a (redundancy) factor of $2 \mathrm{~V} / \mathrm{b}_{0}$. A common choice for the complex Morlet is $V=4$ and $b_{0}=1$, leading to a redundancy factor of 8 , in contrast to redundancies proportional to the number of samples of the time sequence of direct continuous implementations and in particular of the ST.

\section{Group Arrival Determination}

The group arrival determination is performed on the TFR of EGFs, which are the stacked noise cross correlations. Seismological imaging studies need the group arrival times or group velocities (for surface waves) as a function of frequency. We, therefore, transform the time-scale domain PWS into a tf-PWS for the final analysis. The transformation can be performed by just employing an inverse wavelet transform and subsequent forward ST or more directly by employing [40, eq. (22)]. To find the group arrivals, we identify amplitude (or energy) maxima as a function of frequency in the TFR. Ambiguous detections are common for problematic data or cross correlations with a small signal-to-noise ratio (SNR) at certain frequency bands. Different maxima can coexist due to multipathing, scattering, or the presences of other signals and noise. Some of them might be due to fortuitous or accidental summation. As shown later, we reduce signal identification ambiguities by selecting the maxima after a data resampling approach. A welcomed side benefit of this strategy is the robustness assessment of the measurements due to their variability with respect to changes in the database. At any moment, arrival time $t(f)$ can be transformed to group velocities $v_{g}(f)=x / t(f)$ using the travel distance $x$, which equals the interstation distance of the cross correlations.

1) Random Sampling and Subset PWSs: We employ repeatedly the simple random sampling (SRS) strategy [43] to draw $N$ different sets of subsidiary noise cross correlation data bases for $N$ successive tf-PWS analyzes. SRS is the most basic and unbiased sampling procedure. More sophisticated sampling procedures can be employed without any loss of generality. In our implementation, each cross correlation is subjected to

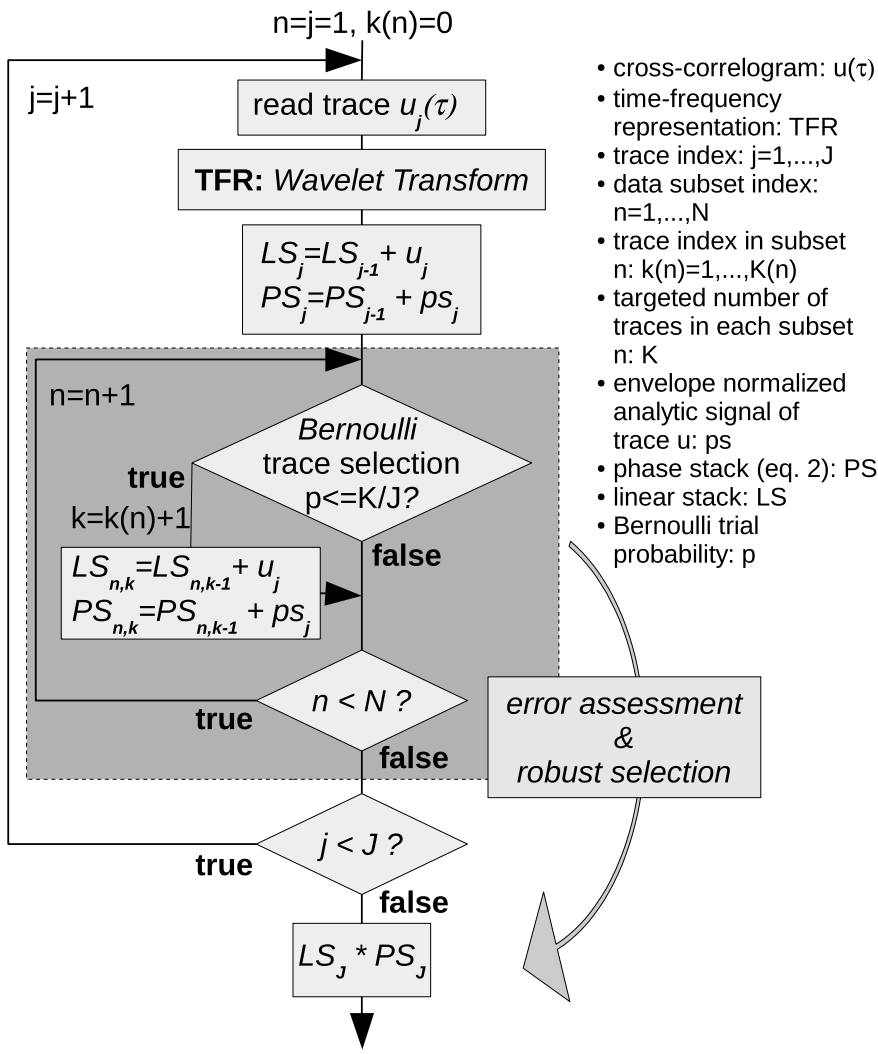

Fig. 1. Flowchart illustrates the decision flow and corresponding actions to build the tf-PWS and the different tf-PWS subsets. The tf-PWS subsets form the base of the robust group arrival extraction.

an independent Bernoulli trial [44], which determines whether a cross correlation becomes part in a subsidiary database. Each cross correlation has an equal probability of being included in a subset. The probability of success is the subset fraction $p=K / J$, where $J$ is the total number of cross correlations (i.e., the population number of the entire database) and $K$ the targeted total number of cross correlations in the drawn final subsets. Note that $K$ should be large enough to guarantee signal extraction in the resulting EGFs.

We construct the different tf-PWS subsets while building the tf-PWS of the entire database, large gray box in the flow diagram of Fig. 1, and LS and PS stand for linear stack and phase stack (2), respectively. All computations can be performed in the time-scale domain. Here, we compute the LSs in the time domain and use their TFR for computing the tf-PWS for each subset.

2) Robust Group Arrivals: We localize the amplitude maxima as a function of frequency in each tf-PWS subset starting from the lowest frequency within a predefined frequency band. The group velocity curve or ridge tracking starts at the lowest frequency and largest energy maximum and progressively goes to higher frequencies by finding the group velocity, which is closest to its previous measure. For each of the $N$ tf-PWS subsets, we determine the group velocities of the four largest maxima within a predefined velocity window and store the value with the smallest velocity jump as a function of frequency. Anomalous velocity jumps are discarded and, in case of spectral holes and temporarily vanishing maxima, the last value is kept as long as the jump is not too large. We 


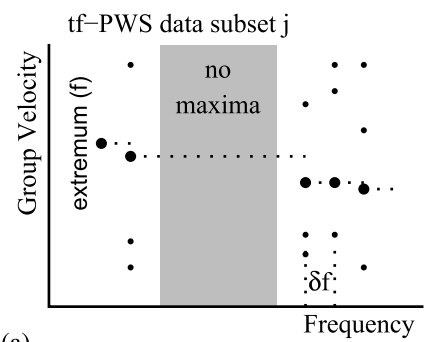

(a)
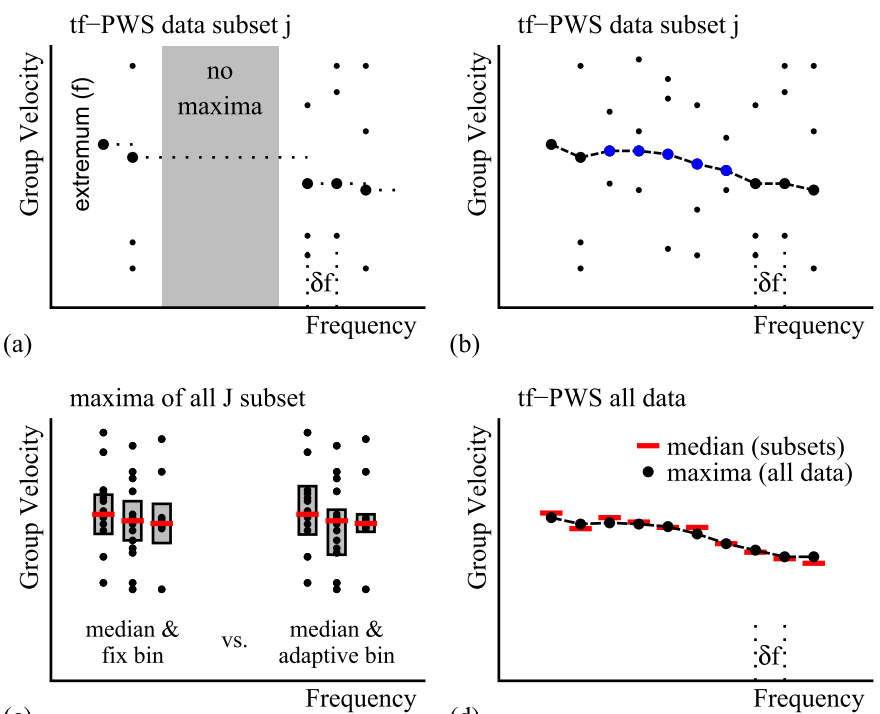

(c)

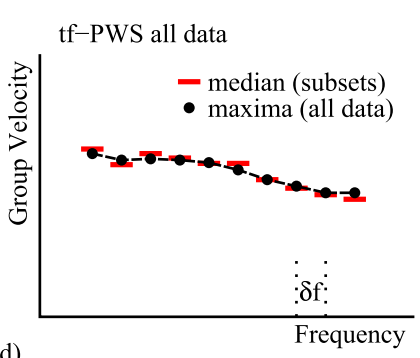

(d)

Fig. 2. Finding robust group arrivals based on repeated detections in tf-PWS data subsets. (a) Black dots are energy maxima for the subset $j$. Bold dots mark selected maxima, tracked starting from the lowest frequency. Gray area represents a spectral hole without maxima. (b) Similar as in (a), but blue dots mark maxima with amplitudes below a predefined threshold value. These values are not considered in the median, but optionally used for the group arrival tracking. (c) Selected maxima from all $J$ subsets (black dots) and their median velocities (red bars). Left and right examples sketch the fix and adaptive bin strategies to count measurements around each median value. (d) Median group arrivals are marked in red and used to find the nearest group arrival (black dots) in the tf-PWS of the entire database. illustrate this procedure in Fig. 2(a) where black dots mark up to four energy maxima per frequency and bold dots mark the selected group velocity at each frequency.

The group velocity ridge is also followed for amplitudes below an amplitude threshold, but the corresponding velocities are only output to the subsequent statistics if the amplitudes are larger than the threshold. This is shown in Fig. 2(b) where the blue dots mark maxima with amplitudes smaller than a threshold. They help bridging weak energy zones but are likely less well constrained and are not kept for the statistics. This way, we propose for each of the $N$ tf-PWS subsets an independent group velocity curve, which can be discontinuous at different frequencies.

Next, we compute the median group velocities as a function of frequency and count the number of successful detections within a small velocity window around the medium group velocity. Alternatively, we determine the amount of detected maxima, which cluster around the median. This last approach helps to improve data adaption, since the maxima detection density can adapt to frequency-dependent resolution. Both strategies are shown in Fig. 2(c). The gray boxes mark the zones for counting the maxima and the red bars mark the median group velocities. If the number of detections is high enough, then the group velocity measure is considered to be robust against data variations and the corresponding final group velocity is measured on the TFR of the tf-PWS for the entire data set. This is shown in Fig. 2(d). The red bars mark the median values and the black dots the nearest energy maxima in the TFR of the tf-PWS of the entire data set.

The robustness of the measurements is estimated from the data subset detection density and through the absolute median
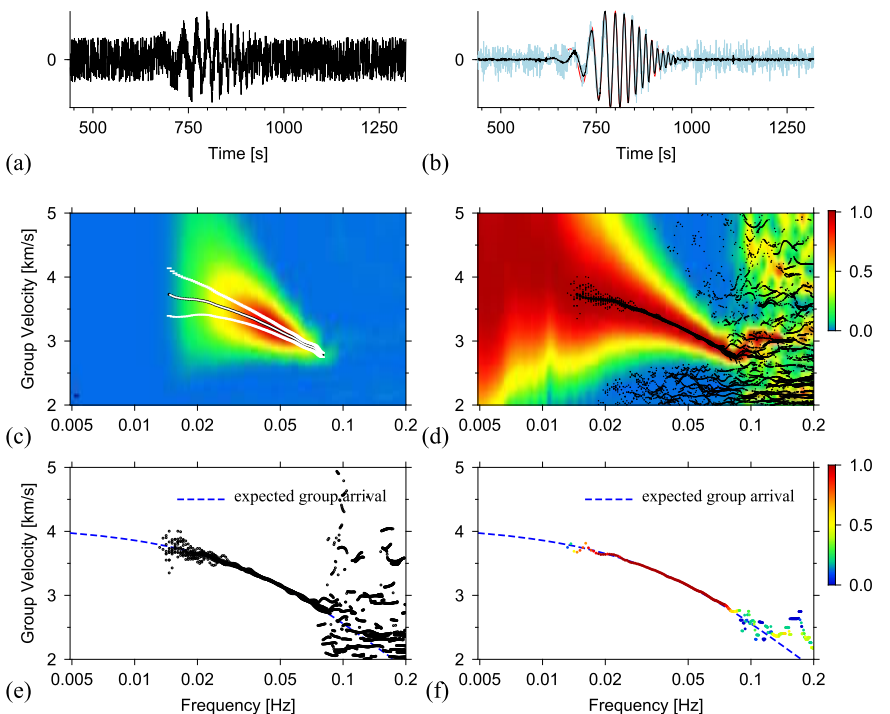

Fig. 3. Theoretical data example. (a) Input data trace. (b) Waveforms for one randomly selected tf-PWS subset (blue line), the tf-PWS of all data (black line), and the chirp function without noise (red line). (c) Contour plot of the TFR of the entire data tf-PWS. The extracted group velocities and 95\% amplitude contours are shown as white lines. (d) Contour plot of the frequency normalized TFR of the entire data tf-PWS. Black dots mark all detected maxima from all subsets. (e) Black dots are the selected maxima for all subsets. The dashed line is the expected group velocity. (f) Colored dots mark the median group velocity for the selected measurements from all subsets. The final measurements are taken from the tf-PWS of all data based on these median velocities. The colors indicate the normalized number of measurements clustered around the median.

deviation of the maxima used to estimate the number of detections. Note that these values are not errors, and they only give confidence into a measure through repeated detection with respect to variations in the database.

3) Numerical Example: For this example, we use a linear chirp function $u(t)=A_{0} e^{-a t^{2}} e^{-i \omega(t) t}$ with $\omega(t)=\omega_{0}+b t$ to obtain a synthetic waveform, which is dispersed in frequency. Here, we use $\omega_{0}=2 \pi 0.04 \mathrm{~Hz}, a=0.0001 \mathrm{~s}^{-2}$, and $b=0.0008 \mathrm{~s}^{-2}$. The corresponding group arrival time can be computed analytically to $\tau_{g}(\omega)=d \phi /(d \omega)=0.5 b(\omega-$ $\left.\omega_{0}\right)^{2} /\left(a^{2}+b^{2}\right)$, where $\phi(\omega)$ is the phase spectrum of $u(t)$, obtained after a Fourier transformation. Fig. 3(a) shows one out of 20 chirp functions contaminated by white noise. In Fig. 3(b), we show the tf-PWS of 20 noise contaminated chirps (black curve), the chirp without any noise contamination (red curve), and the tf-PWS for a subsidiary data set of three traces (blue curve). For this example, we use $N=10$ data subsets and an independent Bernoulli trial sampling probability of $p=0.1$. The corresponding ten subsidiary data sets contain 1-7 traces with a mean and a median of 3.3 and 3 traces, respectively.

Fig. 3(c) shows the amplitude S-spectrum of the tf-PWS of all 20 chirp functions [black curve in Fig. 3(b)]. The amplitude spectrum is normalized to 1 and the color scale is shown to the right. The group arrival time has been transformed to group velocity assuming a propagation distance of $2640 \mathrm{~km}$. The central white line is the extracted group velocity curve and the outer white lines mark the 5\% amplitude decay from the group maximum. The TFR of the same data, but normalized to one per frequency is shown in Fig. 3(d). This normalization is sometimes used to identify and track the group arrival also 

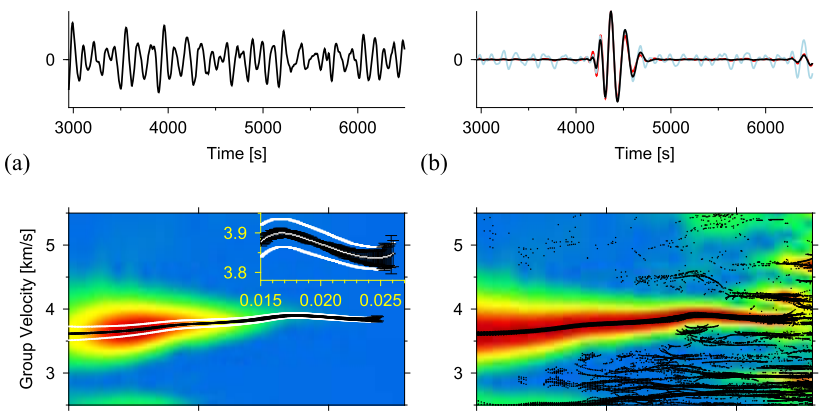

(c) 0.005

\begin{abstract}
0.01
\end{abstract}

\begin{abstract}
0.02
\end{abstract}
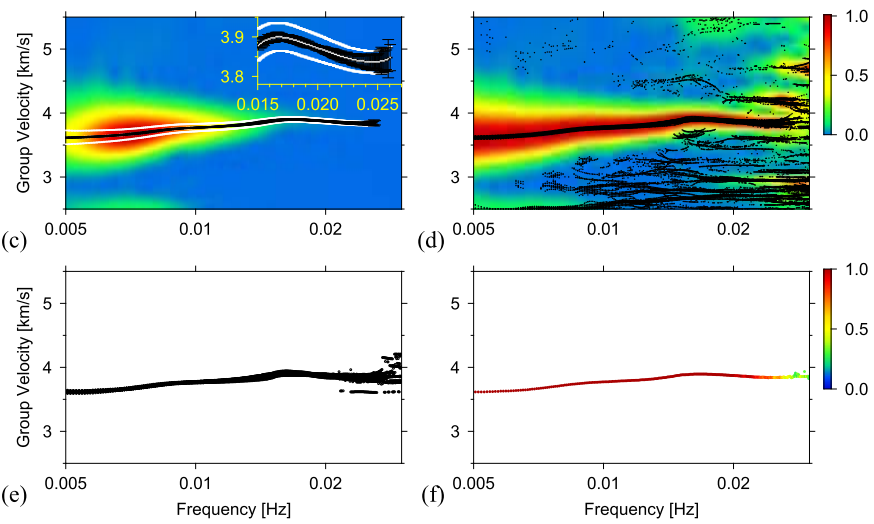

(d) 0.005

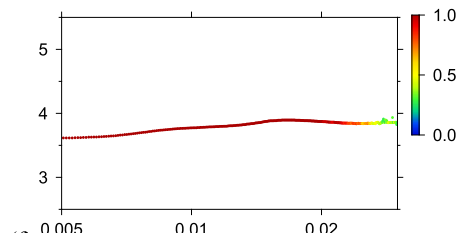

(f) $\begin{array}{lll}0.005 & 0.01 & 0.02 \\ & \text { Frequency [Hz] }\end{array}$

Fig. 4. Field data example using GEOSCOPE stations CAN (Canberra, Australia) and TAM (Tamanrasset, Algeria). Interstation distance is $16.233 \mathrm{~km}\left(145.9^{\circ}\right)$. (a) Randomly selected cross correlation. (b) tf-PWS using all data (black line), the LS of all data (blue line), and a randomly selected subset tf-PWS (red line). (c) Contour plot of the TFR of the tf-PWS for all data [black line in (b)]. The extracted group velocities and 95\% amplitude contours are shown as white lines. The black bars are the absolute median deviations. (d) Contour plot of the frequency normalized TFR of the tf-PWS for all data [black line in (b)]. Black dots mark all detected maxima from all subsets. (e) Black dots are the selected maxima from all tf-PWS subsets. (f) Colored dots mark the median group velocities for the selected measurements from all subsets. The final measurements are taken from the tf-PWS of all data based on these median velocities. The colors indicate the normalized number of measurements clustered around the median.

for small amplitude maxima. The black dots show the up to four largest amplitude maxima per frequency for all subsidiary data sets. Amplitude maxima are defined as maxima whenever there are no larger amplitudes for the previous and next two time samples. In consequence, this is the reason why at lower frequencies, no maxima have been found.

From these maxima, we keep those higher than a threshold amplitude, set at the $20 \%$ of the median amplitude in the TFR of each subset tf-PWS. We further limit the maximum velocity jump to a detected maximum at the nearest lower frequency to less than $0.2 \mathrm{~km} / \mathrm{s}$. Maxima, which satisfy these selection criteria, are plotted as black dots in Fig. 3(e). The blue line marks the expected group velocity curve for all frequencies. Increasing, for instance, the permitted velocity jump from 0.2 to $2 \mathrm{~km} / \mathrm{s}$ manifests in the existence of maxima at a broader group velocity band for frequencies larger than 0.1 Hz. This, however, does not change the final result. For these maxima, we estimate the median group velocity per frequency and count the number of maxima within a $\pm 0.02 \mathrm{~km} / \mathrm{s}$ window. The median group velocity and the number of maxima are shown in Fig. 3(f), where 1 means $100 \%$ of possible detections, i.e., 10 in this example. Finally, we take the final group velocity measurement from the tf-PWS of all traces by choosing the maxima nearest to the median velocities with at least $70 \%$ of possible detections within a $\pm 0.02 \mathrm{~km} / \mathrm{s}$ window. The central white curve of Fig. 3(c) represents the result.

4) Phase Velocity Determination: This publication focuses on the group velocity determination; nevertheless, we mention that the presented strategies can also be employed to measure
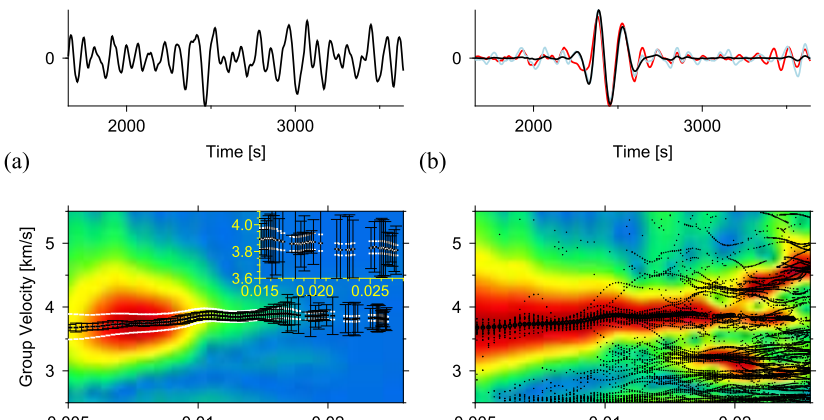

(c) 0.005

0.01

0.02

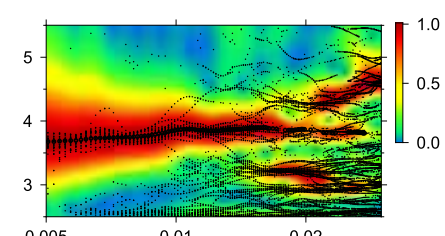

(d) 0.005
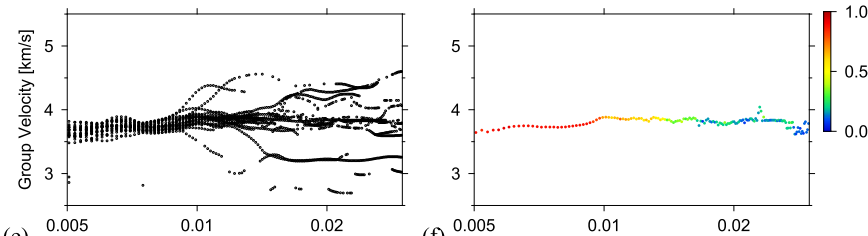

(e) 0.005

(f) 0.005
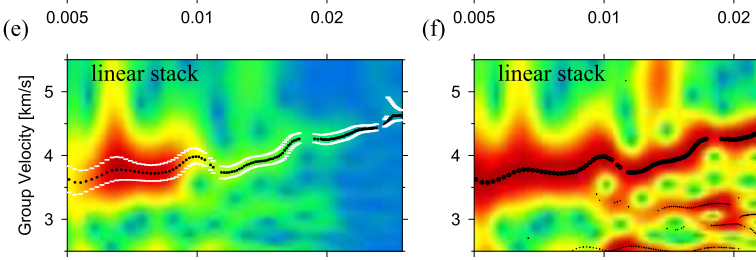

(g) 0.005

Frequency $[\mathrm{Hz}]$

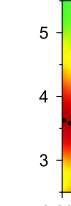

(h) 0.005

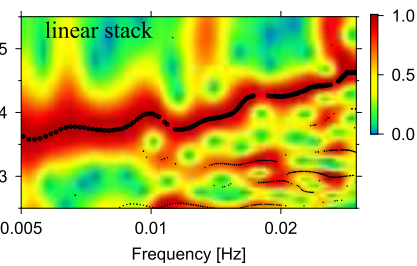

Fig. 5. Field data example using GEOSCOPE stations CLF (Chambon la Foret Observatory, France) and SCZ (Santa Cruz, CA, USA). Interstation distance is $9.100 \mathrm{~km}\left(81.9^{\circ}\right)$. (a)-(f) Similar as in Fig. 4. (g) Contour plot of the TFR of the LS of all data. The black dots mark maxima. (h) Similar as (g) but using the frequency normalized TFR.

phase velocities. For this purpose, one can adopt the strategy by [17] and [45] [their (11) and (7), respectively], who measure phase velocities based on the previously identified group arrivals. Note that the tf-PWS (3) does not alter the phases $\phi\left(t_{g}, \omega\right)$, since the coherence weight $c(\tau, f)$ is a positive real number. The tf-PWS may help identifying the group arrival through attenuation of incoherent signal summation, which translates to the phase velocity estimation.

\section{FIELD DATA EXAMPles}

In this section, we show the group velocity extraction for two seismic station pairs: CAN-TAM and CLF-SCZ. The stations CAN (Canberra, Australia), TAM (Tamanrasset, Algeria), CLF (Chambon la Foret Observatory, France), and SCZ (Chualar Canyon, Santa Cruz, California, USA) are GEOSCOPE stations and their data can be freely downloaded (www.geoscope.ipgp.fr). The vertical components for one year of data were cut into 1-h overlapping, 4-h duration windows, and bandpass filtered (Butterworth, two poles) from 5- to 40-mHz frequency. PCC has been used to compute the cross correlations without any further preprocessing. Classical cross correlations could have been computed, although the correlation approach is not relevant to present the group velocity extraction.

Fig. 4 shows the extraction of the dispersion curve for CAN-TAM. The interstation distance is $16.233 \mathrm{~km}\left(145.9^{\circ}\right)$. A randomly selected PCC is shown in Fig. 4(a). The tf-PWS and LS of all PCCs are shown as black and blue curves in Fig. 4(b). The red curve is a randomly selected subset tf-PWS. For the group velocity extraction, we use a Bernoulli trial 
TABLE I

Variable Description and Values Used for Figs. 4-6. Values Are Flexible, and Different Sets Can Provide Similar Results. The Last Column Contains the Values For the First ITERATIOn With More THAN 70\% SucCess. Numbers IN BRACKETS Are for the Second Iteration AduUstments. Note That Data With DifFerent Characteristics (Frequency Range, EGF Conversion, and Quality) May Need Different Values

\begin{tabular}{|c|c|c|c|c|}
\hline Variable description & $\begin{array}{l}\text { Parameter } \\
\text { in code }\end{array}$ & Fig. 4 & Fig. 5 & Fig. 6 \\
\hline $\begin{array}{l}\text { Probability } p \text { for a trace to be included in a tf-PWS subset (Bernoulli } \\
\text { trial). }\end{array}$ & rpro & 0.5 & 0.3 & $0.3[0.3-0.8]$ \\
\hline Number $N$ of tf-PWS subsets. & nbsmp & 25 & 25 & 25 \\
\hline $\begin{array}{l}\text { Required number of subsets with detections clustered around medium } \\
\text { group velocity ( } 0 \text {-1 with } 1 \text { being } 100 \% \text { ) }\end{array}$ & nugbo & 0.6 & 0.2 & $0.2[0.2-0.8]$ \\
\hline Half width of median group velocity window $(\mathrm{km} / \mathrm{s})$. & ugwin & 0.01 & 0.01 & 0.01 \\
\hline $\begin{array}{l}\text { Data-adaptive median group velocity window. Increase width until } \\
\text { required number of detections or difference between velocity mea- } \\
\text { surements }>\text { specified value }(\mathrm{km} / \mathrm{s}) \text {. }\end{array}$ & gap & 0.2 & 0.2 & $0.2[0.1-0.3]$ \\
\hline Largest permitted velocity jump $(\mathrm{km} / \mathrm{s})$ & dgmax & 0.2 & 0.1 & $0.2[0.1-0.3]$ \\
\hline $\begin{array}{l}\text { Relative amplitude threshold (with respect to median amplitude), i.e., } \\
\text { min. amplitude for a detection. }\end{array}$ & medlim & 0.1 & 0.1 & $0.1[0.05-0.2]$ \\
\hline Group velocity range $(\mathrm{km} / \mathrm{s})$. & grp1,grp2 & $2.5,5.5$ & 2.55 .5 & 2.55 .5 \\
\hline Frequency range $(\mathrm{Hz})$. & $\mathrm{f} 1, \mathrm{f} 2$ & $0.005,0.03$ & $0.005,0.03$ & $0.005,0.03$ \\
\hline Power $\nu$ for the tf-phase coherence (tf-PWS). & wu & 2 & 2 & 2 \\
\hline $\begin{array}{l}2 \sigma \text {-width of f-dependent Gaussian (tf-resolution defined through num- } \\
\text { ber of periods, typically } 2-6 \text { ). }\end{array}$ & cyc & 4 & 4 & 4 \\
\hline
\end{tabular}

probability of $p=0.5$, the number of subsets of $N=25$, and $60 \%$ detection threshold meaning that more than 15 subsets should provide a velocity measure clustered around the median velocity of all subsets. Fig. 4(c) and (d) shows the TFR of the tf-PWS of all data [Fig. 4(b) (black curve)], where amplitudes are normalized to their overall maximum and their maximum per frequency, respectively. The white lines and black bars [Fig. 4(c)] mark the 5\% amplitude decay and the absolute median deviation. As the absolute median deviation is very small in this example, we also show a zoomed-in view of the measurements at high frequencies. The thin white line seen in the center of the inlet is the final measured group velocity. The black dots in Fig. 4(d) mark the detected maxima of all subset tf-PWSs. The selected maxima are shown in Fig. 4(e). Fig. 4(f) shows the corresponding median velocity and number of detections clustered around the median velocity normalized to 1 . This median velocity is used to find the nearest group arrival in the tf-PWS of all data. This example shows a clear group velocity detection.

In full analogy, the results for the station pair CLF-SCZ (interstation distance of $9100 \mathrm{~km}$ or $81.9^{\circ}$ ) are presented in Fig. 5. Here, we used $p=0.3, N=25$, and $20 \%$ detection threshold. It can be seen from Fig. 5 that the final tf-PWS is not as clean as in the previous example and different maxima are detected for different tf-PWS subsets [Fig. 5(d)], specially at frequencies higher than $0.01 \mathrm{~Hz}$. Fig. 5(c) shows that the presented algorithm extracts a group velocity curve, which is equivalent to the one an analyst would have extracted. The absolute median deviations (black vertical bars) reflect the increased ambiguities at the higher frequencies. These ambiguities are also reflected in the decrease of signals clustered around the median [colored points in Fig. 5(f)]. Using a Bernoulli trial probability of $p=0.6$ and a $30 \%$ detection threshold yields the same dispersion with filled gaps and decreased absolute median deviations. The new probability $p$

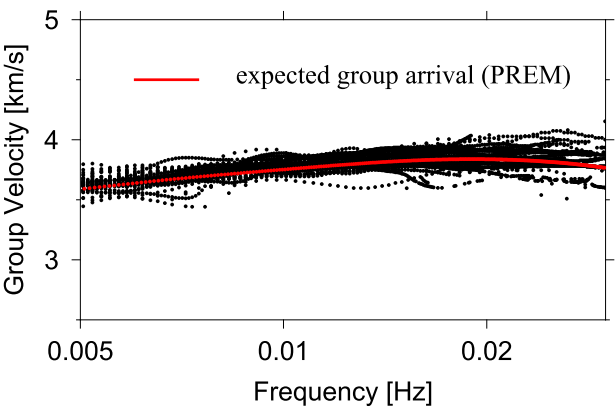

Fig. 6. Dispersion curves for 50 GEOSCOPE station pairs (black dots) and expected group velocity for the PREM model (red line).

increases the number of traces in each tf-PWS subset, which decreases the detection variability and, consequently, the absolute median deviations. The absolute median deviations depend on the parameters, but used as a function of frequency they point to the robustest measurements. Furthermore, as long as the algorithm proposes a median group velocity closer to the correct group velocity maximum than to any other maximum, the correct velocity will be extracted from the tf-PWS of all data. This last step does not depend on the parameters of the algorithm. A fine tuning of parameters is to control the measurement of group velocities at frequencies with a more complex TFR due to the presence of other dominant signals. It permits to add or remove measurements for an optimum dispersion curve extraction. Furthermore, Fig. 5(g) and (h) shows the TFR of the LS of all data in analogy to Fig. 5(c) and (d). It can be seen that the TFR of the LS is much noisier than the TFR of the tf-PWS and yields to a wrong group velocity estimation.

Fig. 6 shows the automatically extracted dispersion curves for 50 GEOSCOPE station pairs (black dots) and the expected velocities for the spherical symmetric Preliminary reference Earth model PREM [46]. For less than 30\% of the dispersion curves, the algorithm was ran a second time with adjusted 
variables to extract group velocities in areas with increased ambiguities. The spread around the reference is mainly due to the different paths of the globally distributed station pairs and seismic inhomogeneities. This type of data is used for imaging the seismic structure.

In Table I, we summarize the parameters used in our field data examples. The values are flexible and variations in the results should manifest in areas with increased ambiguities first. Other data with other characteristics (frequency range, SNR, EGF conversion, preprocessing, among others) may require different values. For instance, a slower convergence to a robust surface-wave signal may need larger $p$ values to increase the number of traces in each subset.

\section{Conclusion And Discussion}

Our algorithm extracts robust dispersion measurements from surface waves emerging from the stacks of noise cross correlations. The approach uses the stacks of subsidiary data sets to help finding the dispersion measurements in the stacks of the entire data set. Data resampling strategies are often used in different applications to assess the robustness of measurements against data variability and are often used to evaluate measurement errors. In our approach, we use resampling to guide the search of group arrivals rather than to evaluate a final measurement. A side product of the resampling strategy is that one can use the variability of measurements to assign a robustness or consistency measure, for instance, the median of absolute median deviations or the standard deviation. The variability does not give the error in the data, but it provides a relative measure on how much the group velocity estimation is consistent.

Another distinction of our method consists in using the tf-PWS [34], [36] to attenuate incoherent noise considering the coherence in the time-frequency domain. This approach is data adaptive due to a time-frequency coherence analysis and, therefore, suited to deal with nonstationary signals and noise. The main purpose is to attenuate the contribution of incoherent signals in the noise correlation stacks. Other independent approaches to measure group velocity are mainly based on MFT [3], [4], [47] and use other cleaning or quality criteria to guarantee the correct measurement of group arrivals. Other quality criteria are based on SNR, agreement to smooth and continuous spline fit group velocity curves [18], and antidispersion or phase-matched filters [48], which compress the dispersed waveforms to clean them from unrelated energy before applying an inverse phase-match filter to uncompress the waveforms. The way we clean the stacks from incoherent signals is completely data adaptive. The seismic attribute used, phase coherence, does not depend on a model and is obtained from the individual constituents of the data stack rather than from the final stack itself, i.e., prestack information rather than poststack information. The PWS has been used before in many different applications (see [49]-[53] as examples from 2015) to enhance small coherent signals and in analogy is suited to measure group velocities [54], [55] robustly. It has further been shown in [55] (their Fig. 1) that robust dispersion curves can be obtained from less data when the tf-PWS approach is being used rather than a conventional stack. This faster convergence to a robust dispersion curve or structural response means that less data are needed for imaging studies and that a higher time resolution can be achieved in monitoring surveys.

The different variables, which guide the decision strategy, can be changed and adjusted for fine tuning. For a robust detection, however, empirical variables are quickly found, so that this approach becomes useful for semiautomated detections in large data sets. The task of controlling the extracted data is not taken by the algorithm. Our method has been tested with theoretical and field data. This method is already operational and being used in different studies (see [55] for a global ambient noise tomography study).

\section{ACKNOWLEDGMENT}

The authors would like to thank the Editor and two anonymous referees for handling and reviewing this paper.

\section{REFERENCES}

[1] J. Oliver, "A summary of observed seismic surface wave dispersion," Bull. Seismol. Soc. Amer., vol. 52, no. 1, pp. 81-86, 1962.

[2] R. L. Kovach, "Seismic surface waves and crustal and upper mantle structure," Rev. Geophys., vol. 16, no. 1, pp. 1-13, Feb. 1978.

[3] A. Dziewonski, S. Bloch, and M. Landisman, "A technique for the analysis of transient seismic signals," Bull. Seismol. Soc. Amer., vol. 59 no. 1, pp. 427-444, 1969.

[4] A. L. Levshin, V. F. Pisarenko, and G. A. Pogrebinsky, "On a frequencytime analysis of oscillations," Ann. Geophys., vol. 28, no. 2, pp. 211-218, 1972.

[5] M. Cara, "Filtering of dispersed wavetrains," Geophys. J. Int., vol. 33, no. 1, pp. 65-80, Jul. 1973.

[6] D. R. Russell, R. B. Herrmann, and H.-J. Hwang, "Application of frequency variable filters to surface-wave amplitude analysis," Bull. Seismol. Soc. Amer., vol. 78, no. 1, pp. 339-354, 1988.

[7] A. Levshin, L. Ratnikova, and J. Berger, "Peculiarities of surface-wave propagation across central Eurasia," Bull. Seismol. Soc. Amer., vol. 82, no. 6, pp. 2464-2493, Dec. 1992.

[8] T. Yamada and K. Yomogida, "Group velocity measurement of surface waves by the wavelet transform," J. Phys. Earth, vol. 45, no. 5, pp. 313-329, 1997.

[9] M. H. Ritzwoller and A. L. Levshin, "Eurasian surface wave tomography: Group velocities," J. Geophys. Res., Solid Earth, vol. 103, no. B3, pp. 4839-4878, Mar. 1998.

[10] C. Ammon, Notes on Seismic Surface-Wave Processing, Part I, Group Velocity Estimation. Saint Louis University, Version 3.9.0. Surface Wave Multiple Filter Analysis Software Documents, 2001.

[11] R. Herrmann and C. Ammon, "Computer programs in seismologysurface waves, receiver functions and crustal structure," Dept. Earth Atmos. Sci., Saint Louis Univ., St. Louis, MO, USA, Tech. Rep., 2002.

[12] T. Lay and T. C. Wallace, Modern Global Seismology, vol. 58. San Francisco, CA, USA: Academic, 1995.

[13] S. Stein and M. Wysession, An Introduction to Seismology, Earthquakes, and Earth Structure. Hoboken, NJ, USA: Wiley, 2009.

[14] P. M. Shearer, Introduction to Seismology. Cambridge, U.K.: Cambridge Univ. Press, 2009.

[15] N. M. Shapiro and M. Campillo, "Emergence of broadband Rayleigh waves from correlations of the ambient seismic noise," Geophys. Res. Lett., vol. 31, no. 7, p. L07614, Apr. 2004.

[16] K. G. Sabra, P. Gerstoft, P. Roux, W. Kuperman, and M. C. Fehler, "Surface wave tomography from microseisms in Southern California," Geophys. Res. Lett., vol. 32, no. 14, p. L14311, Jul. 2005.

[17] G. D. Bensen et al., "Processing seismic ambient noise data to obtain reliable broad-band surface wave dispersion measurements," Geophys. J. Int., vol. 169, no. 3, pp. 1239-1260, Jun. 2007.

[18] C. A. Dalton, J. B. Gaherty, and A. M. Courtier, "Crustal VS structure in northwestern Canada: Imaging the Cordillera-craton transition with ambient noise tomography," J. Geophys. Res., Solid Earth, vol. 116, no. B12, p. B12315, Dec. 2011.

[19] E. Saygin and B. L. N. Kennett, "Crustal structure of Australia from ambient seismic noise tomography," J. Geophys. Res., Solid Earth, vol. 117, no. B1, p. B01304, Jan. 2012. 
[20] M. Pilz, S. Parolai, M. Picozzi, and D. Bindi, "Three-dimensional shear wave velocity imaging by ambient seismic noise tomography," Geophys. J. Int., vol. 189, no. 1, pp. 501-512, Apr. 2012.

[21] H. Kao et al., "Ambient seismic noise tomography of Canada and adjacent regions: Part I. Crustal structures," J. Geophys. Res., Solid Earth, vol. 118, no. 11, pp. 5865-5887, Nov. 2013.

[22] A. Curtis, P. Gerstoft, H. Sato, R. Snieder, and K. Wapenaar, "Seismic interferometry-turning noise into signal," Leading Edge, vol. 25, no. 9, pp. 1082-1092, 2006.

[23] O. Lobkis and R. Weaver, "On the emergence of the Green's function in the correlations of a diffuse field," J. Acoust. Soc. Amer., vol. 110, no. 6, pp. 3011-3017, 2001.

[24] A. Derode et al., "Recovering the Green's function from field-field correlations in an open scattering medium (L)," J. Acoust. Soc. Amer. vol. 113, no. 6, p. 2973, Feb. 2003.

[25] K. Wapenaar, "Retrieving the elastodynamic Green's function of an arbitrary inhomogeneous medium by cross correlation," Phys. Rev. Lett., vol. 93, no. 25, pp. 254301-1-254301-4, Dec. 2004.

[26] R. Snieder, "Extracting the Green's function from the correlation of coda waves: A derivation based on stationary phase," Phys. Rev. E, vol. 69, no. 4, p. 046610, Apr. 2004.

[27] P. Roux, K. G. Sabra, W. A. Kuperman, and A. Roux, "Ambient noise cross correlation in free space: Theoretical approach," J. Acoust. Soc. Amer., vol. 117, no. 1, pp. 79-84, 2005.

[28] K. Wapenaar, E. Slob, and R. Snieder, "Unified Green's function retrieval by cross correlation," Phys. Rev. Lett., vol. 97, no. 23, p. 234301, Dec. 2006.

[29] R. Snieder, K. Wapenaar, and U. Wegler, "Unified Green's function retrieval by cross-correlation; connection with energy principles," Phys. Rev. E, vol. 75, no. 3, p. 036103, Mar. 2007.

[30] K. D. Koper, B. de Foy, and H. Benz, "Composition and variation of noise recorded at the Yellowknife seismic array, 1991-2007," J. Geophys. Res., Solid Earth, vol. 114, no. B10, p. B10310, Oct. 2009.

[31] K. D. Koper, K. Seats, and H. Benz, "On the composition of Earth's short-period seismic noise field," Bull. Seismol. Soc. Amer., vol. 100, no. 2, pp. 606-617, 2010 .

[32] M. Schimmel, E. Stutzmann, F. Ardhuin, and J. Gallart, "Polarized Earth's ambient microseismic noise," Geochem., Geophys., Geosyst., vol. 12, no. 7, p. Q07014, Jul. 2011.

[33] E. Stutzmann, F. Ardhuin, M. Schimmel, A. Mangeney, and G. Patau, "Modelling long-term seismic noise in various environments," Geophys. J. Int., vol. 191, no. 2, pp. 707-722, Nov. 2012.

[34] M. Schimmel and J. Gallart, "Frequency-dependent phase coherence for noise suppression in seismic array data," J. Geophys. Res., vol. 112, no. B4, p. B04303, Apr. 2007.

[35] W. E. Medeiros, M. Schimmel, and A. F. do Nascimento, "How much averaging is necessary to cancel out cross-terms in noise correlation studies?" Geophys. J. Int., vol. 203, no. 2, pp. 1096-1100, 2015.

[36] M. Schimmel, E. Stutzmann, and J. Gallart, "Using instantaneous phase coherence for signal extraction from ambient noise data at a local to a global scale," Geophys. J. Int., vol. 184, no. 1, pp. 494-506, Jan. 2011.

[37] M. Schimmel, "Phase cross-correlations: Design, comparisons, and applications," Bull. Seismol. Soc. Amer., vol. 89, no. 5, pp. 1366-1378, 1999.

[38] M. Schimmel and H. Paulssen, "Noise reduction and detection of weak, coherent signals through phase-weighted stacks," Geophys. J. Int., vol. 130, no. 2, pp. 497-505, 1997.

[39] R. G. Stockwell, L. Mansinha, and R. P. Lowe, "Localization of the complex spectrum: The $S$ transform," IEEE Trans. Signal Process., vol. 44, no. 4, pp. 998-1001, Apr. 1996.

[40] S. Ventosa, C. Simon, M. Schimmel, J. Dañobeitia, and A. Mànuel, "The $S$-transform from a wavelet point of view," IEEE Trans. Signal Process., vol. 56, no. 7, pp. 2771-2780, Jul. 2008.

[41] P. E. T. Jorgensen and M.-S. Song, "Comparison of discrete and continuous wavelet transforms," in Computational Complexity, R. A. Meyers, Ed. New York, NY, USA: Springer, 2012, pp. 513-526.

[42] S. Mallat, A Wavelet Tour of Signal Processing: The Sparse Way, 3rd ed. San Diego, CA, USA: Academic, 2009.

[43] W. G. Cochran, Sampling Techniques. Hoboken, NJ, USA: Wiley, 1977.

[44] A. Papoulis, Probability and Statistics. Englewood Cliffs, NJ, USA: Prentice-Hall, 1990.

[45] F.-C. Lin, M. P. Moschetti, and M. H. Ritzwoller, "Surface wave tomography of the western United States from ambient seismic noise: Rayleigh and Love wave phase velocity maps," Geophys. J. Int., vol. 173, no. 1, pp. 281-298, Apr. 2008.
[46] A. M. Dziewonski and D. L. Anderson, "Preliminary reference Earth model," Phys. Earth Planetary Interiors, vol. 25, no. 4, pp. 297-356, Jun. 1981.

[47] R. B. Herrmann, "Some aspects of band-pass filtering of surface waves," Bull. Seismol. Soc. Amer, vol. 63, no. 2, pp. 663-671, 1973.

[48] A. L. Levshin and M. H. Ritzwoller, "Automated detection, extraction, and measurement of regional surface waves," Pure Appl. Geophys., vol. 158 , no. 8 , pp. $1531-1545,2001$

[49] J. M.-C. Adam and B. Romanowicz, "Global scale observations of scattered energy near the inner-core boundary: Seismic constraints on the base of the outer-core," Phys. Earth Planetary Interiors, vol. 245, pp. 103-116, Aug. 2015.

[50] N. A. Blom, A. Deuss, H. Paulssen, and L. Waszek, "Inner core structure behind the PKP core phase triplication," Geophys. J. Int., vol. 201, no. 3, pp. $1657-1665,2015$.

[51] S. Ventosa and B. Romanowicz, "Extraction of weak $P c P$ phases using the slant-stacklet transform-I: Method and examples," Geophys. J. Int., vol. 201, no. 1, pp. 207-223, 2015.

[52] Y. Almeida, J. Julià, and A. Frassetto, "Crustal architecture of the Borborema province, NE Brazil, from receiver function CCP stacks: Implications for Mesozoic stretching and Cenozoic uplift," Tectonophysics, vol. 649, pp. 68-80, May 2015.

[53] L. Waszek, C. Thomas, and A. Deuss, "PKP precursors: Implications for global scatterers," Geophys. Res. Lett., vol. 42, no. 10, pp. 3829-3838, May 2015.

[54] R. C. Dias, J. Julià, and M. Schimmel, "Rayleigh-wave, group-velocity tomography of the Borborema province, NE Brazil, from ambien seismic noise," Pure Appl. Geophys., vol. 172, no. 6, pp. 1429-1449, Jun. 2015

[55] A. Haned, E. Stutzmann, M. Schimmel, S. Kiselev, A. Davaille, and A. Yelles-Chaouche, "Global tomography using seismic hum," Geophys. J. Int., vol. 204, no. 2, pp. 1222-1236, Feb. 2016.

Martin Schimmel received the Diplom-Geophysiker degree in geophysics from the Karlsruhe Institute of Technology, Karlsruhe, Germany, in 1992 and the Ph.D. degree from Universiteit Utrecht, Utrecht, The Netherlands, in 1997.

From 1997 to 2001, he was a Post-Doctoral Researcher with the Department of Geophysics, Institute of Astronomy, Geophysics and Atmospheric Sciences, University of São Paulo, São Paulo, Brazil. From 2001 to 2008, he was also a Scientific Researcher with the Institute of Earth Sciences Jaume Almera-CSIC, Barcelona, Spain, where he has been a Scientific Staff Member, since 2008. His research interests include seismic signal detection and identification, seismic interferometry, ambient noise studies, and seismic tomography.

Dr. Schimmel was a recipient of the Marie Curie Individual Fellowship at the European Commission's Fifth Framework Programme and the Ramon y Cajal Fellowship at the Spanish Ministry of Education and Science.

Eleonore Stutzmann received the Dipl.Ing. degree from the École et Observatoire des Sciences de la Terre, Strasbourg, France, in 1990, and the Ph.D. degree from the University of Paris 7, Paris, France, in 1993.

From 1993 to 1994, she was a Post-Doctoral Researcher with Utrecht University, Utrecht, The Netherlands. Since 1994, she holds the permanent research position with the Institut de Physique du Globe de Paris (IPGP), Paris. From 2006 to 2015, she has been the Director of Global Seismological Network with GEOSCOPE (France). She is currently a Professor with IPGP and Head of the Seismology Group. Her research interests include seismic noise: modeling the broadband seismic noise, investigating sources of microseisms and hum, and using noise for tomography, landslides and icequakes, ocean bottom seismology, and mantle tomography and geodynamics.

Sergi Ventosa was born in Vilafranca del Penedès, Spain. He received the Engineering degrees in electronics and telecommunication engineering from the Polytechnic University of Catalonia (UPC), Barcelona, Spain, in 1999 and 2002, respectively, and the Ph.D. degree in electronic engineering from Marine Technology Unit, UPC, Barcelona, in 2010, focused on the topic of seismic signal processing.

From 2010 to 2011, he was a Post-Doctoral Researcher with IFP Energies nouvelles, Rueil Malmaison, France. He was with the Institut de Physique du Globe de Paris, Paris, France, from 2011 to 2015. He is currently with the Institute of Earth Sciences Jaume Almera-CSIC, Barcelona. His research interests include seismic signal processing, deep earth structure, denoising, and array processing. 